\title{
AMALGAMATIONS OF LATTICE ORDERED GROUPS
}

BY

\author{
KEITH R. PIERCE
}

\begin{abstract}
The author considers the problem of determining whether certain classes of lattice ordered groups (l-groups) have the amalgamation property. It is shown that the classes of abelian totally ordered groups (ogroups) and abelian $l$-groups have the property, but that the class of $l$-groups does not. However, under certain cardinality restrictions one can find an $l$-group which is the "product" of $l$-groups with an amalgamated subgroup whenever (a) the $l$-subgroup is an Archimedian 0 -group, or (b) the $l$-subgroup is a direct product of Archimedian $o$-groups and the $l$-groups are representable. This yields a new proof that any $l$-group is embeddable in a divisible $l$-group, and implies that any $l$-group is embeddable in an $l$-group in which any two positive elements are conjugate.
\end{abstract}

1. Introduction. It is known that the class of groups [12] and the class of lattices [6, Theorem 3.5 , p. 204] have the amalgamation property, so it is natural to ask whether various classes of lattice ordered groups have the amalgamation property. For the classes of abelian (totally) ordered groups (o-groups) and abelian lattice ordered groups ( $l$-groups) we readily obtain affirmative answers in $\$ 2$ from the knowledge that these classes contain relative injectives. In $\$ 3$ we show that the property fails for the class of all l-groups.

$\$ 5$ is concerned with an attempt to determine some amalgams of l-groups which are embeddable in an l-group. N. R. Reilly [10, Theorem 2.1, p. 8] proved that any amalgam of l-groups, in which the $l$-subgroup is in the center of the containing $l$ groups, is embeddable in an l-group. Under some cardinality restrictions, we prove, using a technique suggested by E. Weinberg, that any amalgam in which the l-subgroup is an Archimedian o-group is embeddable in an l-group. As a corollary, any amalgam of representable l-groups, in which the l-subgroup is a direct product of Archimedian o-groups, is embeddable in an $l$-group. The first result implies an alternate proof-similar in technique to the proof of the classic analogue for groupsof the result of Holland's [5, Theorem 5, p. 405], that any l-group can be embedded in a divisible l-group. Finally, as a corollary to the proof of the first result, we get that an $l$-group can be embedded in an l-group of the same cardinality in which any two positive elements are conjugate.

Notation. All groups are written multiplicatively, and all maps are written on

Presented to the Society, August 30, 1971; received by the editors November 18, 1970 and, in revised form, November 4, 1971. $20 \mathrm{~F} 25$.

AMS (MOS) subject classifications (1970). Primary 06A55, 06A60, 08A25; Secondary

Key words and phrases. Amalgamation property, amalgams of lattice ordered groups, lattice ordered groups, totally ordered groups, extensions of lattice ordered groups. 
the right. $\Pi_{\alpha} M_{\alpha}$ denotes the (unrestricted) direct product of the sets $M_{a}$, and elements of $\Pi_{\alpha} M_{a}$ are written as functions $k$, where $k(\alpha) \in M_{a}$. If $\left\{S_{a} \mid \alpha \in A\right\}$ is a collection of mutually disjoint ordered sets indexed by an ordered set $A$, then $\bigcup_{a}^{*} S_{a}$ denotes the lexicographic union of the sets $S_{a}$, where the orders on each $S_{\alpha}$ are extended to the union by defining $s<t$ whenever $s \in S_{\alpha}, t \in S_{\beta}$, and $a<\beta$. If $S$ is an ordered set and $T \subseteq S$ then $\operatorname{conv}_{S}(T)=\{x \mid a \leq x \leq b$ for some $a \in T, b \in T\}$. If $s \in S$ then $s<T$ means $s<t$ for all $t \in T$.

Any ordinal number $\gamma$ can be written uniquely as $\delta+n$, where $\delta$ is a limit ordinal and $n$ is a finite ordinal, denoted by $n(\gamma) . \mu$ is a regular cardinal if whenever a set of ordinals less than $\mu$ is cofinal in $\mu$, then the set has cardinality $\mu$. $\mu^{*}$ denotes the cardinality of the set of all subsets of $\mu$ of cardinality less than $\mu$. We note that

$$
\mu^{*}=\sum_{\eta<\mu} \mu^{\eta}=\sum_{\eta<\mu} 2^{\eta}
$$

where $\eta$ is a cardinal number.

If $x$ is a member of some l-group, then $|x|=x \vee x^{-1}$; otherwise $|x|$ denotes the cardinality of $x$.

2. Abelian l-groups. We assume familiarity with abstract algebras for the first part of this section. An isomorphism from an algebra $G$ to an algebra $H$ which is not necessarily onto will be called an embedding. The minimal subalgebra of $H$ is the intersection of all subalgebras of $H$. Let $\mathcal{K}$ be a class of similar algebras. $\mathcal{K}$ has the amalgamation property if, whenever $G, H$, and $K$ are in $\mathcal{K}$ and $\sigma_{1}: G \rightarrow$ $H$ and $\sigma_{2}: G \rightarrow K$ are embeddings, there is an $L$ in $\mathcal{K}$ with embeddings $\eta_{1}: H \rightarrow$ $L$ and $\eta_{2}: K \rightarrow L$ such that $\sigma_{1} \eta_{1}=\sigma_{2} \eta_{2}$. A sequence $\left\langle G, H, K, \sigma_{1}, \sigma_{2}\right\rangle$ is an amalgam of $G$ in $H$ and $K$ if $\sigma_{1}: G \rightarrow H$ and $\sigma_{2}: G \rightarrow K$ are embeddings. An amalgam is embeddable in $L$ if there embeddings $\eta_{1}: H \rightarrow L$ and $\dot{\eta}_{2}: K \rightarrow L$ such that $\sigma_{1} \eta_{1}=\sigma_{2} \eta_{2}$. In this case $L$ is called an amalgamation of $G$ in $H$ and $K$.

It is well known [9, p. 108] that if a class $\mathcal{K}$ of algebras is closed under taking direct products, if all minimal subalgebras are mutually isomorphic, and if $\mathcal{K}$ is injectively complete (i.e., every member of $\mathcal{K}$ has an extension which is injective for $\mathcal{K}$ ) then $\mathcal{K}$ has the amalgamation property. Unfortunately, the classes of abelian o-groups and abelian l-groups do not have injectives ([11], [13]). Weinberg [15] and Ribenboim have noted, however, that a weaker version of injectivity does almost as well in many instances. An algebra $L$ in $\mathcal{K}$ is $\mathcal{K}_{a}$-saturated for $\mathcal{K}$ if, whenever $G$ and $H$ are in $\mathcal{K},|H|<\aleph_{a}$, and $\sigma: G \rightarrow H$ and $\tau: G \rightarrow L$ are embeddings, then there is an embedding $\eta: H \rightarrow L$ such that $\sigma \eta=\tau$. $L$ is $\aleph_{a}$-injective for $\mathcal{K}$ if, in the above definition, $\tau$ and $\eta$ are required only to be homomorphisms. $\mathcal{K}$ is relatively saturated complete (relatively injectively complete) if every algebra in $K$ has $\boldsymbol{\aleph}_{a}$ saturated ( $\boldsymbol{\aleph}_{\alpha}$-injective) extensions for arbitrarily large $\alpha$. The above theorem now 
holds if $\mathcal{K}$ is only relatively injectively complete. An easy argument shows a related result:

Theorem 2.1. If all the minimal subalgebras of algebras in $\mathcal{K}$ are mutually isomorphic and $\mathcal{K}$ is relatively saturated complete, then $\mathcal{K}$ bas the amalgamation property.

Alling [1] has shown the existence of abelian o-groups which are $\aleph_{a}$-saturated for this class-namely divisible o-groups which are $\eta_{a}$-sets-and this leads immediately to the

Corollary 2.2. The class of abelian o-groups bas the amalgamation property.

Now an $\boldsymbol{\aleph}_{a}$-saturated abelian 0 -group is $\boldsymbol{\aleph}_{a}$-injective for the class of abelian l-groups: this follows easily from Lemma 6.1 of [15], which states that if $G$ is an l-subgroup of the abelian l-group $H$, then any epimorphism from $G$ to an o-group can be extended to an epimorphism from $H$ to a larger o-group. Since a direct product of $\aleph_{\alpha}$-injectives is $\aleph_{\alpha}$-injective, and since every abelian $l$-group is a subdirect product of abelian o-groups, we have the

Theorem 2.3.(1) The class of abelian l-groups is relatively injectively complete, and bence has the amalgamation property.

3. l-groups. If $S$ is an ordered set let $A(S)$ be the group of order-preserving permutations (o-permutations) of $S . A(S)$ is an l-group if it is given the pointwise order. Holland [5, Theorem 2, p. 401] has shown that every $l$-group $G$ can be embedded in some $A(S)$. We will use his particular embedding, henceforth called a Holland embedding, described as follows: A prime subgroup of $G$ is a convex $l$-subgroup $P$ of $G$ with the property that $a \wedge b=1$ implies $a \in P$ or $b \in P$. In [5] it is shown that for every $1 \neq g \in G$ there is a prime subgroup $P$ of $G$ missing $g$, and that $G / P$, under the lattice ordering induced by $G$, is totally ordered. Suppose $\left\{P_{\alpha} \mid \alpha \in A\right\}$ is a collection of prime subgroups of $G$ such that $\bigcap_{a} P_{\alpha}=1$. If $A$ is totally ordered in some fashion, then $G$ can be embedded in $A\left(\bigcup_{\alpha}^{*} G / P_{\alpha}\right)$ by letting each element of $G$ act by right multiplication on each coset in $S$. Furthermore, the set of prime subgroups can be chosen judiciously enough so that $|A| \leq|G|$. We note that, if $P$ is a prime subgroup of $G$, the right regular representation of $G$ is a homomorphism of $G$ into $A(G / P)$.

Theorem 3.1. The class of l-groups does not have the amalgamation property.

Proof. Let $H$ be the group with generators $a, b$, and $c$ subject to the laws $a b=b a, a c=c b$, and $b c=c a$ (cf. [2, p. 18]). $H$ becomes an l-group if we define $a^{m} b^{n} c^{p} \geq 1$ if and only if $p>0$, or $p=0$ and $m \geq 0$ and $n \geq 0$. Let $G$ be the l-subgroup of $H$ generated by $a, b$, and $c^{2}$; as a group, $G$ is the direct product of the

(1) The author wishes to thank the referee for simplifying the proof of this theorem. 
cyclic groups $A, B$ and $C$ generated by $a, b$, and $c^{2}$ respectively. Let $\sigma_{1}: G \rightarrow$ $H$ be the inclusion map. $A$ and $B$ are disjoint prime subgroups of $G$, so there is a Holland embedding $\sigma_{2}: G \rightarrow A(S)$, where $S=G / A \cup^{*} G / B$. Let $k$ be the element of $A(S)$ defined by

$$
(A x) k=A x, \quad(B x) k=B x c^{2} .
$$

Finally, let $K$ be the $l$-subgroup of $A(S)$ generated by $G \sigma_{2} \cup\{k\}$. Note that $b \sigma_{2} \wedge$ $k=1$.

We show that the amalgam $\left\langle G, H, K, \sigma_{1}, \sigma_{2}\right\rangle$ is never embeddable in an l-group. Suppose, by way of contradiction, that $L$ is an l-group and $\eta_{1}: H \rightarrow L$ and $\eta_{2}: K$ $\rightarrow L$ are embeddings such that $\sigma_{1} \eta_{1}=\sigma_{2} \eta_{2}$. Let $P$ be the smallest convex $l$-subgroup of $K$ containing $b \sigma_{2}$ and $k^{\prime}=k\left(c^{-2} \sigma_{2}\right)$. Evidently, $P=\{x \in K|| x|\leq| y \mid$ for some element $y$ of the subgroup of $K$ generated by $k^{\prime}$ and $\left.b \sigma_{2}\right\}$. Hence, since $k^{\prime}$ and $b \sigma_{2}$ leave fixed the element $B$ of $S$, so does each element of $P$. Thus $a \sigma_{2}$ $\notin P$, since $(B)\left(a \sigma_{2}\right)=B a \neq B$. Thus let $N$ be a convex l-subgroup of $L$ which includes $P \eta_{2}$ and is maximal with respect to not containing $a \sigma_{2} \eta_{2} . N$ is a prime subgroup of $L[5$, Lemma 8], so let $\pi$ be the natural homomorphism of $L$ into $A(L / N)$.

Now, in $L / N, N c \eta_{1}<N(a c) \eta_{1} \leq N c^{2} \eta_{1}$, for replacing the inequality by an equality would imply that $a \sigma_{2} \eta_{2}=a \eta_{1} \in N$, contradicting the definition of $N$. Thus $\left(N c \eta_{1}\right)\left(b \sigma_{2} \eta_{2} \pi\right)=N(c b) \eta_{1}=N(a c) \eta_{1}>N\left(c \eta_{1}\right)$. Since $b \sigma_{2} \wedge k=1$, then $b \sigma_{2} \eta_{2} \pi \wedge$ $k \eta_{2} \pi=1$ in $A(L / N)$ and hence $\left(N c \eta_{1}\right)\left(k \eta_{2} \pi\right)=N c \eta_{1}$. Since $k^{\prime} \eta_{2} \in N$, we thus have the following string of inequalities: $\left(N c \eta_{1}\right)\left(k \eta_{2} \pi\right)=N c \eta_{1}<N c^{2} \eta_{1}=N c^{2} \sigma_{2} \eta_{2}=$ $N\left(k^{\prime} \eta_{2}\right)\left(c^{2} \sigma_{2} \eta_{2}\right)=N k \eta_{2}=(N)\left(k \eta_{2} \pi\right) \leq\left(N c \eta_{1}\right)\left(k \eta_{2} \pi\right)$, which is impossible. Thus the amalgamation $L$ never existed in the first place.

4. The main lemma. We generalize the definition of $\eta_{a}$-sets (cf. Hausdorff [4]) as follows: $T$ is an a-splitting subset of an ordered set $S$ if $A \subseteq S, B \subseteq S, A<B$,

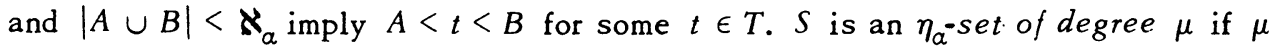
is a cardinal and $S$ is the disjoint union of a collection of $\alpha$-splitting subsets $\left\{S_{i} \mid i<\mu\right\}$. Evidently $\eta_{a}$-sets of degree 1 are $\eta_{a}$-sets. Hausdorff [4] showed that all $\eta_{a}$-sets of cardinality $\aleph_{a}$ are isomorphic, and this generalizes to

Theorem 4.1. If $S=\bigcup S_{i}$ and $T=\bigcup T_{i}(i<\mu)$ are $\eta_{a}$-sets of degree $\mu$ and cardinality $\aleph_{a}$, then there is an isomorphism $\eta: S \rightarrow T$ such that $S_{i} \eta=T_{i}$ for all $i<\mu$.

Proof. Let $\left\{s_{\gamma} \mid \gamma<\boldsymbol{\aleph}_{a}\right\}$ and $\left\{t_{\gamma} \mid \gamma<\boldsymbol{N}_{\alpha}\right\}$ be well-orderings of $S$ and $T$. Suppose $\left\{\eta_{\gamma} \mid \gamma<\boldsymbol{\aleph}_{\alpha}\right\}$ is a tower of 1-1 order-preserving functions satisfying, for each $\gamma<$ $\aleph_{a}$

(a) ${ }_{\gamma}:\left\{s_{\beta} \mid \beta<\gamma\right\} \subseteq$ Domain $\left(\eta_{\gamma}\right) \subseteq s$,

(b) ${ }_{\gamma}:\left\{t_{\beta} \mid \beta<\gamma\right\} \subseteq$ Range $\left(\eta_{\gamma}\right) \subseteq T$, and

(c) ${ }_{\gamma}: s_{\beta} \in S_{i} \cap \operatorname{Domain}\left(\eta_{\gamma}\right)$ if and only if $s \eta_{\gamma} \in T_{i}$. 
Evidently $\eta=\bigcup_{y} \eta_{y}$ is the desired isomorphism, so it will suffice to construct this tower. Let $\delta<\boldsymbol{N}_{\alpha}$, and suppose the tower $\left\{\eta_{\gamma} \mid \gamma<\delta\right\}$ has been constructed satisfying, for all $\gamma<\delta$, the axioms (a) $)^{-(c)_{\gamma}}$, and the additional condition

(d) ${ }_{\gamma}:\left|\eta_{\gamma}\right|<\aleph_{\alpha}$.

If $\delta$ is a limit ordinal then $\eta_{\delta}=\bigcup_{\gamma<\delta} \eta_{\gamma}$ satisfies $(a)_{\delta}-(\mathrm{d})_{\delta}$. If $\delta=\gamma+1$, then we can extend $\eta_{\gamma}$ to a 1-1 order-preserving function $\eta_{\delta}$ satisfying (c) $\delta$ and (d) $\delta$ so that the domain of $\eta_{\delta}$ contains the element of $S$ of least subscript not in the domain of $\eta_{\gamma}$, and the range of $\eta_{\delta}$ contains the element of $T$ of least subscript not in the range of $\eta_{\gamma}$. Hence also $(a)_{\delta}$ and $(b)_{\delta}$ are satisfied. Thus by transfinite induction the entire sequence can be constructed.

Later we will need to recognize $\eta_{a}$-sets of degree $\mu$ and cardinality $\boldsymbol{\aleph}_{a}$ as the union of a certain tower of ordered sets. The following lemma can be considered as a generalization of Satz 1 of [4]. Its proof is straightforward.

Lemma 4.2 Let $\boldsymbol{N}_{a}$ be a regular cardinal and let $\left\{U_{\gamma} \mid \gamma<\aleph_{\alpha}\right\}$ be a tower of ordered sets satisfying

(i) $U_{\gamma}$ is the disjoint union of a collection of subsets $\left\{S_{i}(\gamma) \mid i<\mu\right\}$ each of cardinality at most $\boldsymbol{N}_{a}$, and $\left|u_{\gamma}\right|<\boldsymbol{N}_{a}$,

(ii) $\gamma<\gamma^{\prime}$ implies $S_{i}(\gamma) \subseteq S_{i}\left(\gamma^{\prime}\right)$, and

(iii) for each $\gamma<\mathbb{N}_{\alpha}$, if $A \subseteq U_{\gamma}, B \subseteq U_{\gamma}, A<B$, and $|A \cup B|<\mathcal{N}_{a}$, then for all $i<\mu$ there exist $\gamma^{\prime}>\gamma$ and $s \in S_{i}\left(\gamma^{\prime}\right)$ such that $A<s<B$.

Then $S=\bigcup_{i} S_{i}$ is an a-set of degree $\mu$ and cardinality $\aleph_{\alpha}$, where $S=$ $\cup_{\gamma} U_{\gamma}$ and $S_{i}=\bigcup_{\gamma} S_{i}(\gamma)$.

$A \subseteq S$ is said to be a positive (negative) interval of $g \in A(S)$ if $A=$ $\operatorname{conv}_{s}\left\{s g^{n} \mid n \in Z\right\}$ for some $s$ such that $s<s g(s g<s)$. A zero interval of $g$ is a maximal convex set of fixed points of $g$. Let $\mathcal{P}(\mathrm{g}), \Re(g), \mathcal{M}(\mathrm{g}), \mathcal{Z}(\mathrm{g})$, and $\mathcal{G}(\mathrm{g})$ denote respectively the set of all positive, negative, nonzero, zero, and all intervals of $g . g(g)$ is a partition of $S$ into convex subsets and hence is naturally ordered.

Main Lemma 4.3. If $\boldsymbol{\aleph}_{a}$ is a regular cardinal such that $\boldsymbol{N}_{a}^{*}=\boldsymbol{\aleph}_{a}$, $M$ is an $\mathbf{l}$ group, and $|M| \leq \mathcal{N}_{\alpha}$, then there is an embedding $\phi: M \rightarrow A(S)$ for some $\eta_{a}$-set $S$ of cardinality $\boldsymbol{N}_{a}$ sucb that for each $1 \neq g \in M$

(i) $g \phi$ bas bounded support,

(ii) each zero interval of $g \phi$ is an $\alpha$-set, and

(iii) if $g^{*}(g \phi)$ and $\mathcal{Z} *(g \phi)$ are gotten from $\mathcal{G}(g \phi)$ and $\mathcal{Z}(g \phi)$ by throwing out the least and greatest zero intervals of $g \phi$, then $g *(g \phi)=\mathbb{M}(g \phi) \cup \mathcal{Z}^{*}(g \phi)$ is an $\eta_{a}$-set of degree 2 and cardinality $\boldsymbol{\aleph}_{a}$.

The proof occurs after the next two lemmas.

In the next lemma we use a slight generalization of the wreath product of $l$ - 
groups of o-permutations, first discussed by Lloyd [7, p. 40]. Let $M$ be an $l$-subgroup of $A(S) . \gamma$ is a $M$-orbit of $S$ if, for some $s \in S, \gamma=\{s g \mid g \in M\}$. Let $\Gamma$ be the set of $M$-orbits of $S$ (a partition of $S$ ) and let $\gamma(s)$ denote the M-orbit to which $s$ belongs. For each $\gamma \in \Gamma$ let $T_{\gamma}$ be an ordered set, let $M^{\gamma}$ be an l-subgroup of $A\left(T_{\gamma}\right)$, and let $S^{*}=\left\{(t, s) \mid s \in S, t \in T_{\gamma(s)}\right\}$ where $(t, s) \leq\left(t^{\prime}, s^{\prime}\right)$ if and only if $s<s^{\prime}$, or $s=s^{\prime}$ and $t \leq t^{\prime}$. The orbital wreath product $W$ of $M$ with $\left\{M^{\gamma} \mid \gamma \in \Gamma\right\}$ is the $l$-subgroup of $A\left(S^{*}\right)$ consisting of all ordered pairs $(k, g)$ where $g \in M$ and $k \in \Pi_{s} M^{\gamma(s)}$, and where each ordered pair acts on $S^{*}$ by the rule $(t, s)(k, g)=$ $(t(k(s)), s g)$. The proof that $W$ is an $l$-subgroup of $A\left(S^{*}\right)$ is routine, for the group and lattice operations are identical with those in the wreath product of two o-permutation groups, and we display these operations here: For $k \in \Pi_{s} M^{\gamma(s)}$ and $g \in M$ define $k^{g}(s)=k(s g)$ and let $i(s)=1$. Then $(i, 1)$ is the identity of $W$, $(k, g)(m, b)=\left(k m^{8}, g b\right)$ and $(i, 1) \vee(k, g)=(n, 1 \vee g)$, where

$$
n(s)= \begin{cases}1 & \text { if } s g<s, \\ 1 \vee k(s) & \text { if } s g=s, \\ k(s) & \text { if } s<s g .\end{cases}
$$

Let $M$ be an l-group and $S \subseteq S^{\prime}$. The embedding $\phi: M \rightarrow A\left(S^{\prime}\right)$ is said to expand the embedding $\psi: M \rightarrow A(S)$ if each o-permutation $g \phi$ is an extension of $g \psi$. In this case there is a natural embedding of $\mathbb{M}(g \psi)$ into $\mathbb{M}(g \phi)$ taking $\mathcal{P}(g \psi)$ into $\mathcal{P}(g \phi)$ and $\pi(g \psi)$ into $\pi(g \phi)$. In general $\mathcal{E}(g \psi)$ is not naturally embedded in $Z(g \phi)$.

Lemma 4.4. An embedding $\psi: M \rightarrow A(S)$ can be expanded to $\phi: M \rightarrow A\left(S^{*}\right)$ so that.

(i) $\left|S^{*}\right| \leq \max \{|M|,|S|\}$,

(ii) if $\mathfrak{Q}$ is a nonempty collection of nonzero intervals of $g \psi$ and $s=$ $\inf _{S}(\cup \mathfrak{Q})\left(S=\sup _{S}(\bigcup \mathfrak{A})\right)$, then $s<B<\cup \mathbb{Q}(\cup \mathfrak{G}<B<s)$ for some nonzero interval $B$ of $g \phi$, and

(iii) eacb zero interval of $g \psi$ is a subset of some zero interval of $g \phi$.

Proof. Assume $M \subseteq A(S)$, and let $\Gamma$ be the set of $M$-orbits of $S$. For each $\gamma \epsilon$ $\Gamma$ fix $0_{\gamma} \in \gamma$ and let $\{\langle r s\rangle \mid r \in \gamma, s \in \gamma\}$ be a subset of $M$ such that $r\langle r s\rangle=s$, $\langle r s\rangle^{-1}=\langle s r\rangle$ and $\langle r s\rangle\langle s t\rangle=\langle r t\rangle$. Define l-ideals $L_{\gamma}$ and $U_{\gamma}$ of the stabilizer subgroup $M_{0_{\gamma}}$ as follows: If $0_{\gamma}$ is the least element of $S$ put $L_{\gamma}=M$, otherwise let $L_{\gamma}$ be the set of $g \in M$ fixing all elements of some proper interval $\left[a, 0_{\gamma}\right]$. If $0_{\gamma}$ is the greatest element of $S$ put $U_{\gamma}=M$, otherwise let $U_{\gamma}$ be the set of $g \in M$ fixing all elements of some proper interval $\left[0_{\gamma}, a\right]$. Using Holland embeddings, consider $M_{0 y} / L_{\gamma}$ and $M_{0 y} / U_{\gamma}$ to be l-subgroups of $A\left(R_{\gamma}\right)$ and $A\left(S_{\gamma}\right)$ respectively, where $\left|R_{\gamma}\right|$ and $\left|S_{\gamma}\right|$ are at most $|M|$. Let $T_{\gamma}=R_{\gamma} \cup^{*}\left\{u_{\gamma}\right\} \cup^{*} S_{\gamma}$ and let $\phi_{\gamma}$ be the homomorphism of $M_{0 \gamma}$ into $A\left(T_{\gamma}\right)$ defined by letting $g \phi_{\gamma}$ act like $L_{\gamma} g$ on $R_{\gamma}$, like $U_{\gamma} g$ on $S_{\gamma}$, and like the identity on $u_{\gamma}$. Embed $S$ in $S^{*}=\{(t, s) \mid s \in S, t \in$ $\left.T_{\gamma(s)}\right\}$ by identifying $s$ with $\left(u_{\gamma(s)}, s\right)$. Finally, let $\phi$ map each $g \in M$ to $\left(g^{*}, g\right)$ in the orbital wreath product of $M$ with $\left\{M_{0 \gamma} \mid \gamma \in \Gamma\right\}$, where 


$$
g^{*}(s)=\left(\left\langle 0_{\gamma(s)} s\right\rangle_{g}\left\langle s g 0_{\gamma(s)}\right)\right) \phi_{\gamma(s)} .
$$

First we remark that $g^{*}(s)$ is determined by the action of $g$ on sufficiently small neighborhoods of $s$; i.e., if $g$ and $b$ act alike on some interval $[a, b]$ for which $a<s<b$, then $g^{*}(s)=b^{*}(s)$. For then $g b^{-1}$ acts like the identity on $[a, b]$. Conjugating by $\left\langle s 0_{\gamma(s)}\right\rangle(\gamma=\gamma(s))$ results in $g^{\prime}=\left\langle 0_{\gamma} s\right\rangle g b^{-1}\left\langle s 0_{\gamma}\right\rangle$ acting like the identity on an interval about $0_{\gamma}$, whence $g^{\prime} \in L_{0 \gamma} \cap M_{0 \gamma}$. This implies that $1=$ $\left.g^{\prime} \phi_{\gamma}=\left(\left\langle 0_{\gamma} s\right\rangle_{g}\left\langle s g 0_{\gamma}\right\rangle\right) \phi_{\gamma}\left[\left\langle 0_{\gamma} s\right\rangle b\left\langle s b 0_{\gamma}\right\rangle\right) \phi_{\gamma}\right]^{-1}=g^{*}(s) b^{*}(s)^{-1}$, as desired.

The proof that $\phi$ is a group homorphism depends on the identity $(g h)^{*}=g^{*}\left(b^{*}\right)^{g}$, which is straightforward and therefore omitted. We prove that $\phi$ is a lattice homomorphism. Since $1 \vee g \phi=(n, 1 \vee g)$, where $k$ is replaced by $g^{*}$ in equation (S), it suffices to show that $(1 \vee g) *(s)=n(s)$ for all $s \in S$. Letting $\gamma=\gamma(s)$, we consider three cases: First suppose that $s g<s$. Then $1 \vee g$ is the identity on the interval $\left[s g, s g^{-1}\right]$ about $s$, whence by the above remark $(1 \vee g) *(s)=1=n(s)$. Next, suppose that $s g=s$. Then also $s=s(1 \vee g)$ and we calculate directly that $(1 \vee g) *(s)=$ $\left(\left\langle 0_{\gamma} s\right\rangle(1 \vee g)\left\langle s(1 \vee g) 0_{\gamma}\right\rangle\right) \phi_{\gamma}=\left(1 \vee\left\langle 0_{\gamma} s\right\rangle_{g}\left\langle s g 0_{\gamma}\right\rangle\right) \phi_{\gamma}=1 \vee g^{*}(s)=n(s)$. Finally, suppose that $s<s g$. Then $g$ and $1 \vee g$ act alike on the interval $\left[s g^{-1}, s g\right]$ about $s$, so $(1 \vee g)^{*}(s)=g^{*}(s)=n(s)$. Now $\phi$ expands the inclusion map on $M$, since $s(g \phi)=$ $\left(u_{\gamma(s)}, s\right)\left(g^{*}, g\right)=\left(u_{\gamma(s)} g^{*}(s), s g\right)=\left(u_{\gamma(s)}, s g\right)=\left(u_{\gamma(s g)}, s g\right)=s g$. This also shows that $\phi$ is one-to-one.

(i) is obvious since $|\Gamma| \leq|S|$.

To show (ii), let $\varnothing \neq \mathfrak{Q} \subseteq \mathbb{M}(g)$ and suppose $s=\inf _{s}(\bigcup \mathfrak{Q})$. Then $s g=s$, for otherwise $s$ would be in the interior of some $A \in \mathbb{M}(g)$. Furthermore, $g$ moves some element of each proper interval $[s, t]$. Conjugating by $\left\langle s 0_{\gamma}\right\rangle$, where $\gamma=\gamma(s)$, we have that $g^{\prime}=\left\langle 0_{\gamma} s\right\rangle_{g}\left\langle s g 0_{\gamma}\right\rangle$ is in $M_{0 y}$ but not in $U_{\gamma}$, so there is some $B^{\prime} \epsilon$ $\pi\left(g^{\prime} \phi_{\gamma}\right)$ such that $u_{\gamma}<B^{\prime}$. Thus $B=\left\{(t, s) \mid t \in B^{\prime}\right\}$ is a nonzero interval of $g \phi$ such that $s<B<\bigcup \mathfrak{Q}$.

To prove (iii), we must show that if $D$ is a zero interval of $g$ and $s^{*} \epsilon$ conv $_{s^{*}}(D)$, then $s^{*}(g \phi)=s^{*}$. Let $s^{*}=(t, s)$, and suppose $c \leq s^{*} \leq d$ for some $c$, $d \in D$. Then $c \leq s \leq d$, which implies that $s g=s$, so it suffices to show that $\operatorname{tg}(s)=t$. If $c<s<d$ then $g$ acts like the identity on an interval about $s$, whence by the above note $g^{*}(s)=1$. If $c=s=d$ then $t=u_{\gamma(s)}$, so by definition $\operatorname{tg}^{*}(s)=t$. If $c=s<d$ then $u_{\gamma(s)} \leq t$ and $g$ acts like the identity on the interval $[s, d]$. Thus, $\left\langle 0_{\gamma(s)} s\right\rangle_{g}\left\langle s g 0_{\gamma(s)}\right\rangle^{\gamma(s)}$ acts like the identity on an interval above $0_{\gamma(s)}$. This implies that $g^{*}(s)$ acts like the identity on $S_{\gamma(s)}$, which implies that $\operatorname{tg} *(s)=t$. If $c<s=$ $d$ the proof is similar, so in any case $\operatorname{tg}^{*}(s)=t$.

Let $T \subseteq S \subseteq S^{\#}$. The element $s^{\prime} \in S^{\#}$ is said to sever $S$ above $T$ (below $T$ ) if $T<s^{\prime}<s\left(s<s^{\prime}<T\right)$ whenever $s \in S$ and $T<s \quad(s<T)$. If $T$ has no greatest element and $s^{\prime}$ is a unique element of $S^{\#}$ severing $S$ above $T$, then $s^{\prime}$ is the supremum of $T$ in $S^{\#}$.

Lemma 4.5. If $|S| \leq \aleph_{a}$, then an embedding $\psi: M \rightarrow A(S)$ can be expanded to $\phi: M \rightarrow A\left(S^{\#}\right)$ so that 
(i) $\left|S^{\#}\right| \leq \boldsymbol{N}_{a}^{*}$,

(ii) if $T \subseteq S$ and $|T|<\aleph_{a}$ then there are unique elements of $S^{\#}$ which sever $S$ above and below $T$, and

(iii) each zero interval of $g \psi$ is a subset of some zero interval of $g \phi$.

Proof. Let $S^{\#}$ be the union of $S$ with the set of all ordered pairs $(A, B)$, where $A \cup B=S, A<B$, and either $A$ has a cofinal subset of cardinality less than $\aleph_{a}$, or $B$ has a coinitial subset of cardinality less than $\aleph_{a}$. Extend the order on $S$ to $S^{\#}$ by defining

$$
\begin{aligned}
s<(A, B) & \text { if } s \in A, \\
(A, B)<s & \text { if } s \in B, \text { and } \\
(A, B) \leq\left(A^{\prime}, B^{\prime}\right) & \text { if } A \subseteq A^{\prime} \text { and } B^{\prime} \subseteq B
\end{aligned}
$$

(cf. [3, p. 118]). Finally, extend each $g \psi$ to $g \phi$ by defining $(A, B)(g \phi)=(A(g \psi)$, $B(g \psi))$. Evidently $\phi$ expands $\psi$, and the other properties follow routinely.

Proof of (4.3). First using a Holland embedding, and then applying (4.4) and (4.5), we can construct a tower of ordered sets $\left\{S_{\gamma} \mid \gamma<\aleph_{\alpha}\right\}$ and a sequence of embeddings $\phi_{\gamma}: M \rightarrow A\left(s_{\gamma}\right)\left(\gamma<\boldsymbol{N}_{\alpha}\right)$ such that for each $\gamma$

(a) $\left|S_{\gamma}\right| \leq K_{\alpha}$,

(b) $\phi_{\gamma}$ expands $\phi_{\delta}$ for each $\delta<\gamma$,

(c) if $\gamma$ is a nonzero limit ordinal, then $S=\bigcup_{\delta<\gamma} S_{\delta}$, and, for each $g \in M$, $g \phi_{\gamma}=\bigcup_{\delta}<\gamma g \phi_{\delta}$,

(d) if $\gamma=\delta+1, n(\delta)$ is even, $T \subseteq S_{\delta}$, and $|T|<\aleph_{\alpha}$, then $S_{\gamma}$ contains unique elements severing $S_{\delta}$ above and below $T$,

(e) if $\gamma=\delta+1, n(\delta)$ is odd, $\varnothing \neq \mathbb{Q} \subseteq \pi\left(g \phi_{\delta}\right)$, and $s=\inf _{s_{\delta}}(\cup \mathfrak{Q})$ $\left(s=\sup _{s}(\mathfrak{U} \mathfrak{Q})\right)$, then $s<B<\bigcup \mathfrak{Q}(\bigcup \mathfrak{Q}<B<s)$ for some $B \in \mathbb{M}\left(g \phi_{\gamma}\right)$, and

(f) if $\delta<\gamma$ and $D \in \mathscr{Z}\left(g \phi_{\delta}\right)$, then $D$ is a subset of some $D^{\prime} \in \mathcal{Z}\left(g \phi_{\gamma}\right)$.

Let $S=U \cup^{*} S^{\prime} \cup^{*} V$, where $S^{\prime}=\cup_{\gamma} S_{\gamma}$ and $U$ and $V$ are $\eta_{a}$-sets of cardinality $\aleph_{a}$. For $g \in M$ let $g \phi$ be the element of $A(S)$ which fixes $U$ and $V$ and acts like $\bigcup_{\gamma} g \phi_{\gamma}$ on $S^{\prime}$. Evidently $\phi$ expands every $\phi_{\gamma}$, and every $g \phi$ has bounded support.

To prove (ii) let $D \in \mathcal{Z}(g \phi)$, and let $D_{1}=D \cap(U \cup V)$ and $D_{2}=D \cap S^{\prime}$. Now if $D_{1} \neq \varnothing$ then $D_{1}$ is all of $U, V$, or $U \cup V$, and hence is an $\eta_{a}$-set of cardinality $\aleph_{a}$. If $D_{2} \neq \varnothing$ then, by (f), $D_{2}=\bigcup D_{\gamma}\left(\delta \leq \gamma<\aleph_{\alpha}\right)$, where $\delta$ is the least ordinal for which $D \cap S_{\delta} \neq \varnothing$, and $D_{\gamma}=D \cap S_{\gamma} \in \mathcal{E}\left(g \phi_{\gamma}\right)$. If $s \in S_{\gamma+1}$ is a unique element severing a subset of $D_{\gamma}$, then $s \in D_{\gamma+1}$. This remark and (d) are enough to show that the sequence $\left\{D_{\gamma} \mid \delta<\gamma<\boldsymbol{N}_{\alpha}\right\}$ satisfies the conditions of (4.2) for $\mu=1$. Thus $D_{1}$, whence also $D$, is an $\eta_{a}$-set of cardinality $\aleph_{a}$. In particular, the case $g=1$ shows that $S$ is an $\eta_{a}$-set of cardinality $\boldsymbol{N}_{a}$.

By (f), whenever $\delta<\gamma, g\left(g \phi_{\delta}\right)$ is naturally embedded in $\mathscr{I}\left(g \phi_{\gamma}\right)$ and $g(g \phi)$ so that $g^{*}(g \phi), \mathbb{R}(g \phi)$, and $\mathcal{Z}^{*}(g \phi)$ are the respective unions of all $g *\left(g \phi_{\gamma}\right), \mathbb{M}\left(g \phi_{\gamma}\right)$, and $\mathcal{Z} *\left(g \phi_{\gamma}\right)$, where $g *\left(g \phi_{\gamma}\right)$ and $\mathcal{Z} *\left(g \phi_{\gamma}\right)$ are gotten by removing from $g\left(g \phi_{\gamma}\right)$ and 
$\mathcal{E}\left(g \phi_{\gamma}\right)$ those zero intervals (if any) which are subsets of the least and greatest zero intervals of $g \phi$.

We will use (4.2) with $\mu=2$ to prove (iii). The only nontrivial part of (4.2) is (iii). Let $\mathfrak{A}, \mathfrak{B} \subseteq \mathfrak{g *}\left(g \phi_{\delta}\right), \mathfrak{Q}<\mathfrak{B}$, and $|\mathfrak{Q} \cup \mathfrak{B}|<\aleph_{a}$. We must find $\gamma>\delta, B \in$ $\pi\left(g \phi_{\gamma}\right)$, and $D \in \mathscr{Z} *\left(g \phi_{\delta}\right)$ so that $\mathfrak{Q}<B, D<\mathfrak{B}$. We consider the cofinal nature of $\mathcal{A}$ and the coinitial nature of $\mathcal{B}$ and investigate separately the four cases which, together with their duals, may occur. We can assume that $n(\delta)$ is even. If $\mathscr{B}$ is nonempty and does not begin with a zero interval of $g \phi_{\delta}$, then there is a coinitial subset $\mathfrak{B}^{\prime}$ of $\mathfrak{B}$ consisting of nonzero intervals. By (d) we find $s=\inf \left(\bigcup \mathfrak{B}^{\prime}\right)$ in $s_{\delta+1}$, and by (e) find $B^{\prime} \in \mathbb{M}\left(g \phi_{\delta+2}\right)$ such that $s<B^{\prime}<\cup \mathfrak{B}^{\prime}$. By (d) again we find $t=$ $\inf \left(\bigcup \mathfrak{B}^{\prime}\right)$ in $S_{\delta_{+} 3}$, where $s<B^{\prime}<t<\mathfrak{B}^{\prime}$. But $t$ is then in some $D \in \mathcal{Z}^{*}\left(g \phi_{\delta+3}\right)$. Thus $s<B^{\prime}<D<\bigcup \mathfrak{B}^{\prime}$, and therefore $\mathfrak{A}<B<D<\mathfrak{B}$, where $B \in \mathbb{M}\left(g \phi_{\delta+3}\right)$ contains $B^{\prime}$. If $\mathfrak{B}$ begins with a zero interval and $\mathfrak{A}$ ends with a zero interval then by the maximality of such intervals there is a $B \in \Pi\left(g \phi_{\delta}\right)$ between $\mathcal{Q}$ and $\mathfrak{B}$. If we adjoin $B$ to $\mathfrak{B}$, we revert to the previous case. Suppose $\mathscr{A}$ is empty and $\mathfrak{B}$ begins with a zero interval $D^{\prime}$. Then $D^{\prime}$ is not a subset of the least zero interval of $g \phi$, so for some $\beta>\delta$ there is a $B \in \mathbb{M}\left(g \phi_{\beta}\right)$ such that $B<D^{\prime}$. Then working in $S_{\beta}$ and letting $\mathcal{Q}=\{B\}$, we revert to the dual of the first case. Finally, if both $\mathscr{C}$ and $\mathbb{B}$ are empty then we may replace $\beta$ with the set consisting of a single nonzero interval of $g \phi_{\delta}$ and again revert to the first case.

5. Amalgamations of some $l$-subgroups; conjugacy in l-groups.

Theorem 5.1. If $G$ is an Archimedian o-group, then the amalgam $\left\langle G, H, K, \sigma_{1}\right.$, $\sigma_{2}$ ) of l-groups is embeddable in an l-group whenever there is a regular cardinal $\boldsymbol{\aleph}_{a}$ sucb that $\boldsymbol{\aleph}_{a}^{*}=\boldsymbol{\aleph}_{a},|G|<\boldsymbol{\aleph}_{\alpha},|H| \leq \boldsymbol{\aleph}_{a}$, and $|K| \leq \boldsymbol{\aleph}_{\alpha}$.

Remark. E. C. Weinberg first conjectured this theorem and presented to the author an outline of this proof. The author was able to supply the details, which make up the body of the previous section.

Proof. Let $\phi: H \rightarrow A(S)$ and $\eta_{2}: K \rightarrow A(T)$ be as in (4.3). Let $1<g \in G$. By (4.1) there is an isomorphism $\mu: g\left(g \sigma_{1} \phi\right) \rightarrow g\left(g \sigma_{2} \eta_{2}\right)$ carrying positive intervals to positive intervals, and zero intervals to zero intervals.

Any isomorphism $\tau^{\prime}: S \rightarrow T$ induces an isomorphism $\tau: A(S) \rightarrow A(T)$ defined by letting $\left(s \tau^{\prime}\right)(f \tau)=(s f) \tau^{\prime}$. If for all $x \in G, x \sigma_{1} \phi \tau=x \sigma_{2} \eta_{2}$, then putting $\eta_{1}=\phi \tau$, we have embedded the given amalgam in $A(S)$. Thus our task is to find a $\tau^{\prime}$ such that for all $x \in G, s \in S$,

$$
\left(s \tau^{\prime}\right)\left(x \sigma_{2} \eta_{2}\right)=\left[s\left(x \sigma_{1} \phi\right)\right] \tau^{\prime} .
$$

We define $\tau^{\prime}$ separately on each interval $A$ of $g \sigma_{1} \phi$, making sure that $\tau^{\prime}$ is an isomorphism from $A$ to $A \mu$. If $A \in \mathcal{Z}\left(g \sigma_{1} \phi\right)$, this is easy, since $A$ and $A \mu$ are both $\eta_{\alpha}$-sets of cardinality $\aleph_{a}$. If $A \in \mathcal{P}\left(g \sigma_{1} \phi\right)$, fix $s_{0} \in A$ and $t_{0} \in A \mu$. Since $G$ is Archimedian, the map 
$\left[s_{0}\left(x \sigma_{1} \phi\right)\right] \tau^{\prime}=t_{0}\left(x \sigma_{2} \eta_{2}\right)$ is an isomorphism from the $G \sigma_{1} \phi$-orbit of $s_{0}$ to the $G \sigma_{2} \eta$ orbit of $t_{0}$, both coterminal subsets of their respective intervals. $\tau$ remains undefined on subsets of $A$ of the form

$$
\left\{s \in S \mid s_{0}\left(u \sigma_{1} \phi\right)<s<s_{0}\left(v \sigma_{1} \phi\right), u \in U, v \in V\right\}
$$

where $(U, V)$ is a nonvoid Dedekind cut of $G$. We notice that the elements of $G \sigma_{1} \phi$ permute these subsets, and that there is at most one element of $G \sigma_{1} \phi$ which takes one such subset to another. Partition the collection of such subsets into transitivity classes and fix $A_{0}$ in a transitivity class. Since $|G|<\boldsymbol{N}_{\alpha}$, then $A_{0}$ is bounded by small enough subsets so that $A_{0}$ is an $\eta_{a}$-set of cardinality $\boldsymbol{N}_{a}$. Hence let $\tau^{\prime}$ be an isomorphism from $A_{0}$ to the corresponding subset of $A \mu$ (i.e., generated by the same cut of $G$ ). If $A_{1}$ is another set in the transitivity class then let $\tau^{\prime}$ carry $s \in A_{1}$ to $s\left(x^{-1} \sigma_{1} \phi\right) \tau^{\prime}\left(x \sigma_{2} \eta_{2}\right)$ where $x \sigma_{1} \phi$ is the unique element of $G \sigma_{1} \phi$ which takes $A_{0}$ to $A_{1}$. Repeating this process for every transitivity class completes the definition of $\tau^{\prime}$ on $A$ and yields the desired isomorphism.

An l-group is representable if it is the subdirect product of o-groups.

Corollary 5.2. If $G$ is the direct product of Archimedian o-groups $G_{\gamma}(\gamma \in \Gamma)$ and $H$ and $K$ are representable l-groups, then the amalgam $\left\langle G, H, K, \sigma_{1}, \sigma_{2}\right\rangle$ is embeddable in an l-group whenever there is a regular cardinal $\boldsymbol{\aleph}_{\alpha}$ such that $\boldsymbol{N}_{\alpha}^{*}=$ $\aleph_{\alpha},|H| \leq \aleph_{\alpha},|K| \leq \boldsymbol{\aleph}_{\alpha}$, and $\left|G_{\gamma}\right|<\boldsymbol{N}_{a}$ for each $\gamma \in \Gamma$.

Proof. Let $H$ and $K$ be $l$-subgroups, respectively, of $\Pi_{a} H_{\alpha}$ and $\Pi_{\beta} K_{\beta}$ where $H_{\alpha}$ and $K_{\beta}$ are o-groups. For each $\gamma \in \Gamma$ choose $g_{\gamma} \in G$ so that $1<g_{\gamma}(\gamma)$ but $1=$ $g_{\gamma}(\delta)$ whenever $\delta \neq \gamma$. The set of all $g_{\gamma}$ form a mutually disjoint subset of $G^{+}$, and hence when its image in $H$ is projected on $H_{a}$, it remains disjoint. Since $H_{a}$ is totally ordered, then there is at most one $\gamma$ for which $g_{\gamma} \sigma_{1}(\alpha) \neq 1$. Now we adjoin a dummy variable 0 to $\Gamma$, set $H_{\gamma}=\Pi\left\{H_{\alpha} \mid g_{\gamma} \sigma_{1}(\alpha) \neq 1\right\}$ and $H_{0}=\Pi\left\{H_{\alpha} \mid g_{\gamma} \sigma_{1}(\alpha)=1\right.$ for all $\gamma \in \Gamma\}$. The above argument guarantees that none of these products have common factors, hence $H$ is in a unique way an l-subgroup of $\Pi_{\xi} H_{\xi}(\xi \in \Gamma \cup\{0\})$. In a similar fashion we consider $K$ to be an l-subgroup of $\Pi_{\xi} K_{\xi}(\xi \in \Gamma \cup\{0\})$. The natural homomorphisms $\sigma_{1 \gamma}: G_{\gamma} \rightarrow H_{\gamma}$ and $\sigma_{2 \gamma}: G_{\gamma} \rightarrow K_{\gamma}$ are therefore embeddings. By (5.1) there is an l-group $L_{\gamma}$ with embeddings $\eta_{1 \gamma}: H_{\gamma} \rightarrow L_{\gamma}$ and $\eta_{2 \gamma}$ : $K_{\gamma} \rightarrow L_{\gamma}$ such that $\sigma_{1 \gamma} \eta_{1 \gamma}=\sigma_{2 \gamma} \eta_{2 \gamma}$. Let $L_{0}$ be any l-group for which there are embeddings $\eta_{10}: H_{0} \rightarrow L_{0}$ and $\eta_{20}: K_{0} \rightarrow L_{0}$. Finally let $L=\Pi L_{\xi}(\xi \in \Gamma \cup\{0\})$ and let $\eta_{1}: H \rightarrow L$ and $\eta_{2}: K \rightarrow L$ be defined by $\left(b \eta_{1}\right)(\xi)=b(\xi) \eta_{1 \xi}$ and $\left(k \eta_{2}\right)(\xi)$ $=k(\xi) \eta_{2 \xi}$. Since for all $g \in G,\left(g \sigma_{1}\right)(\gamma)=[g(\gamma)] \sigma_{1 \gamma}$, then evidently $\sigma_{1} \eta_{1}=\sigma_{2} \eta_{2}$, so we have embedded the amalgam in $L$.

As an application of these results we consider the problem of embedding an $l$ group in a divisible l-group, solved by Holland [5, Theorem 5, p. 405]. B. H. Neumann [8, Theorem 6.2, p. 11] proved that groups are embeddable in divisible groups, and used the fact that the amalgam of a group, where the subgroup is cyclic, 
is embeddable in a group. (4.1) thus provides a similar proof:

Theorem 4.3 (Holland). An l-group $H$ is embeddable in a divisible l-group whenever there is an uncountable regular cardinal $\boldsymbol{N}_{\alpha}=\boldsymbol{N}_{\alpha}^{*}$ sucb that $|H| \leq \boldsymbol{N}_{a}$.

Proof. Let $1<g \in H=H_{0}$. If $G$ is the l-subgroup of $H$ generated by $g, K$ is the additive ordered group of rational numbers, $\sigma_{1}: G \rightarrow H$ is the inclusion map and $\sigma_{2}: G \rightarrow K$ is any embedding, then by (4.1) we can find an $l$-group $H^{\prime}$ containing $H_{0},\left|H_{0}\right|=\left|H^{\prime}\right|$, and in which for each positive integer $n$ the equation $x^{n}=g$ has a solution. Repeating this procedure successively for each positive element of $H_{0}$ and then taking the direct limit, we obtain an l-group $H_{1}$ containing $H_{0},\left|H_{1}\right|=$ $\left|H_{0}\right|$, in which occur all roots of all positive elements of $H_{0}$. Repeating the whole thing a countably infinite number of times and taking the direct limit, we arrive at an l-group $L$ whose positive cone is a divisible semigroup. This guarantees that $L$ is divisible.

Remark. Weinberg [14, Theorem 3, p. 506] has proved (4.3) without the cardinality restriction.

Looking back at the proof of (4.1), we note that if $S=T$ then $\tau^{\prime} \in A(S)$, and $\tau$ is the inner automorphism of $A(S)$ generated by $\tau^{\prime}$. This enables us to find l-groups with homogeneous positive cones in the following sense:

Corollary 5.2 An l-group $M$ is embeddable in an l-group $L$, of the same cardinality as $M$, in which any two positive elements of $L$ are conjugate, whenever there is an uncountable regular cardinal $\boldsymbol{\aleph}_{a}$ such that $\boldsymbol{\aleph}_{a}^{*}=\boldsymbol{\aleph}_{a}$ and $|M| \leq \boldsymbol{\aleph}_{a}$.

Proof. Let $M=M_{0}$ be embedded in $A(S)$ as in (4.3). By the above remarks any two positive elements of $M_{0}$ are conjugate in $A(S)$. If $M_{1}$ is the l-subgroup of $A(S)$ generated by $M_{0}$ together with one conjugating element for each pair of positive elements of $M_{0}$, then any two positive elements of $M_{0}$ are conjugate in $M_{1}$ and $\left|M_{0}\right|=\left|M_{1}\right|$. Repeating this process a countably infinite number of times, we get a tower of l-groups $M_{0} \subseteq M_{1} \subseteq M_{2} \cdots$ in which $\left|M_{i}\right|=\left|M_{0}\right|$ and any pair of positive elements of $M_{i}$ are conjugate in $M_{i+1}$. Then $L=\bigcup_{i} M_{i}$ is the desired l-group.

Remark. The restriction $\boldsymbol{N}_{a}^{*}=\boldsymbol{N}_{a}$ for arbitrarily large cardinals $\boldsymbol{N}_{a}$ is consistent with and independent of the other axioms of set theory, and is evidently implied by the generalized continuum hypothesis.

\section{BIBLIOGRAPHY}

1. N. L. Alling, On ordered divisible groups, Trans. Amer. Math. Soc. 94 (1960), 498514. MR $25 \# 4013$.

2. L. Fuchs, Partially ordered algebraic systems, Pergamon Press, Oxford; AddisonWesley, Reading, Mass., 1963. MR 30 \#2090.

3. E. Harzheim, Beiträge zur Theorie der Ordnungstypen, insbesondere der $\eta_{a^{-M e n g e n} \text {, }}$ Math. Ann. 154 (1964), 116-134. MR 28 \#5011.

4. F. Hausdorff, Grundzüge der Mengenlehre, Veit, Leipzig, 1964. 
5. C. Holland, The lattice-ordered group of automorphisms of an ordered set, Michigan Math. J. 10 (1963), 399-408. MR 28 \#1237.

6. B. Jónsson, Universal relational systems, Math. Scand. 4 (1956), 193-208. MR 20 \#3091.

7. J. T. Lloyd, Lattice ordered groups and o-permutation groups, Doctoral Thesis, Tulane University, New Orleans, La., 1965.

8. B. H. Neumann, Adjunction of elements to groups, J. London Math. Soc. 18 (1943), 4-11. MR 5, 58.

9. R. S. Pierce, Introduction to the theory of abstract algebras, Holt, Rinehart, and Winston, New York, 1968. MR 37. \#2655.

10. N. R. Reilly, Permutational products of lattice ordered groups (to appear).

11. P. Ribenboim, On ordered modules, J. Reine Angew. Math. 225 (1967), 120-146. MR 34 \#5877.

12. O. Schreier, Die Untergruppen der freier Gruppen, Abh. Math. Sem. Univ. Hamburg 5 (1927), 161-183.

13. D. M. Topping, Some homological pathology in vector lattices, Canad. J. Math. 17 (1965), 411-428. MR 30 \#4700.

14. E. C. Weinberg, Embedding in a divisble lattice-ordered group, J. London Math. Soc. 42 (1967), 504-506. MR 36 \#91.

15. - Relative injectives and universals for categories of ordered structures (to appear). 